\title{
GENERAL WIENER-HOPF OPERATORS
}

\author{
BY \\ ALLEN DEVINATZ AND MARVIN SHINBROT( $\left.{ }^{1}\right)$
}

1. Introduction. Let $L^{2}$ be the usual Lebesgue space with respect to Haar measure on the circle group and $H^{2}$ the Hardy space of elements of $L^{2}$ whose Fourier coefficients vanish on the negative integers. Let $a(\theta)$ be a bounded, measurable, complex-valued function on the circle group and $A$ the operator on $L^{2}$ defined by

$$
(\mathrm{A} f)(\theta)=a(\theta) f(\theta) .
$$

Finally, let $\mathrm{P}$ denote orthogonal projection of $L^{2}$ onto $H^{2}$. What is usually called a Toeplitz operator or a Wiener-Hopf operator is

$$
T_{\mathrm{P}}(\mathrm{A})=\mathrm{PA} \mid H^{2},
$$

or any of a number of operators equivalent to (1.2).

Let $\mathfrak{S}$ be any Hilbert space and $\mathfrak{P}$ any closed, linear subspace of $\mathfrak{S}$. If $A$ is any bounded operator on $\mathfrak{S}$ and $P$ is orthogonal projection on $\mathfrak{P}$, an obvious generalization of $(1.2)$ is

$$
T_{P}(A)=P A \mid \mathfrak{P} .
$$

We call any operator of the form (1.3) a Wiener-Hopf operator or, indifferently, a Toeplitz operator. When we wish to emphasize that we are discussing (1.3) with no restriction on $P$ or on $A$, we call (1.3) a general Wiener-Hopf operator. To distinguish the special case (1.2), we call it the special Wiener-Hopf operator.

There is a large literature on questions relating to the invertibility of the special Wiener-Hopf operator. It turns out that many of the most important results proved in the literature can be extended to apply also to general Wiener-Hopf operators. This was exploited by Shinbrot in [12] and [13] when $A$ is a positive operator. It is our purpose in this paper to continue the analysis begun in [12] and [13], omitting the hypothesis that $A$ is positive. To see the type of result we are able to get at, we state here a necessary and sufficient condition for the invertibility of $T_{\mathrm{P}}(\mathrm{A})$ due to Devinatz ([3]; but see also [17]). Let $H^{\infty}$ denote the set of all essentially bounded elements of $H^{2}$. Then, the result of [3] that we have in mind is this. Suppose that

$$
\text { ess } \inf |a(\theta)|>0 \text {. }
$$

Received by the editors November 1, 1968 and, in revised form, May 8, 1969.

( ${ }^{1}$ ) The first author is supported by NSF Grant GP-7638, the second by NSF Grant GP-8936.

Copyright (C) 1969, American Mathematical Society 
Then, the special Wiener-Hopf operator $T_{\mathrm{P}}(\mathrm{A})$ is invertible if and only if there exists an $h \in H^{\infty}$ such that $1 / h \in H^{\infty}$ and

$$
\operatorname{Re}[a(\theta) h(\theta)] \geqq \delta>0 \quad \text { a.e. }
$$

This theorem should be compared with the following result proved in $\$ 2$ below. Suppose that $A$ is an invertible operator on $\mathfrak{S}$. Then, the general Wiener-Hopf operator $T_{P}(A)$ is invertible if and only if there exists a bounded, invertible operator $H$ taking $\mathfrak{B}$ onto itself such that

$$
\operatorname{Re}(A H f, f) \geqq \delta\|f\|^{2}
$$

for all $f \in \mathfrak{S}$.

The relation between the two theorems is clear. For the special Wiener-Hopf operator, the condition that $A$ be invertible is equivalent to (1.4). If, moreover, we identify $H$ with the operator

$$
(H f)(\theta)=h(\theta) f(\theta),
$$

then the conditions that $h$ and $1 / h$ be in $H^{\infty}$ mean that $H$ is invertible and takes $\mathfrak{P}$ onto itself. Finally, in this case, it is easy to see that (1.5) and (1.6) are equivalent.

Thus our theorem is indeed an extension of the quoted theorem of Devinatz. In one respect, however, our result is less precise than Devinatz's. In our theorem, we prove the existence of an operator $H$ having certain properties, while Devinatz proves the existence of a function $h$ which, ex machina, we identified with $H$ via (1.7). The point is not that we expect $H$ to have the form (1.7) in all cases. Indeed, in general, this form does not make sense, and even when it does, it is in general not true that the operator $H$ will have the form (1.7). This special form of $H$ seems to depend on the special structure of $H^{2}$ and it would be most interesting to be able to abstract just those properties of $H^{2}$ which will yield the special result. However, it should be pointed out that for most applications, the sharper form of $H$ for $H^{2}$ spaces does not yield anything better than the abstract version of the theorem. Indeed, since our methods of proof are constructive, we are in one instance able to get results which it does not seem possible to obtain by the nonconstructive methods used in the proof of the theorem for the special Wiener-Hopf operators. We shall say more about this later.

Very recently, H. R. Pousson [9] extended the theorem of Devinatz to the case where $a(\theta)$ is a finite matrix valued function, and M. Rabindranathan [10] extended it to the case where $a(\theta)$ is a bounded, operator-valued function on a separable Hilbert space. We shall have more to say about the results of these authors in the last section of the paper. In $\S 2$, we generalize these results to the abstract context.

To return to our discussion of Devinatz's theorem, the result of [3] is actually better than we have indicated, for the condition (1.4) need not be built into the hypothesis of the theorem. Indeed, it is shown in [3] that, (1.4) and (1.5) are necessary as well as sufficient for the invertibility of $T_{\mathrm{P}}(\mathrm{A})$. Now, in the general 
context, (1.4) is equivalent to the invertibility of $A$. However, easy examples show that in general $A$ need not be invertible when $T_{P}(A)$ is invertible. However, since this is the case for the special Wiener-Hopf operator, and since our purpose here is to explore abstract generalizations of theorems valid for the special Wiener-Hopf operator, in $\S 3$ we consider an abstract situation in which the invertibility of $T_{P}(A)$ implies that of $A$. We then show that when $A=\mathrm{A}$ and $P=\mathrm{P}$, our abstract result implies (1.4). In addition, $\$ 3$ contains an extension to general Wiener-Hopf operators of the so-called Hartman-Wintner spectral inclusion theorem.

Although we have used the names Wiener and Hopf many times, we have not yet pointed out their main contribution. In a context equivalent to that of $T_{\mathrm{P}}(\mathrm{A})$, they showed [15] that the function $a(\theta)$ could be factored into a product of a function analytic in the unit disk and another function conjugate analytic in the disk. By using these factors, they were able to find a formula for $\left[T_{\mathrm{P}}(\mathrm{A})\right]^{-1}$. The method for solving the equation

$$
T_{\mathrm{P}}(\mathrm{A}) f=g
$$

implied by their formula has become known as the Wiener-Hopf method.

In the general context, we can say that

$$
T_{P}(A) f=g
$$

can be solved by a Wiener-Hopf method if $A$ can be factored in a certain way that allows (1.9) to be solved analogously to (1.8). In $\S 4$, we prove the remarkable result that (1.9) can be solved by a Wiener-Hopf method if and only if $T_{P}(A)$ is invertible.

The proof of the main theorem in $\S 4$ is in principle constructive. We utilize this fact in $\S 5$ to derive a closed form for the solution of (1.9) assuming that $A$ satisfies

$$
\operatorname{Re}(A f, f) \geqq \delta\|f\|^{2}, \quad \delta>0 .
$$

If $A$ does not have this property, a technical difficulty arises in the formula for the solution. We also discuss this matter in $\$ 5$.

From the point of view of applications, one of the most important Wiener-Hopf operators is the operator

$$
\left[T_{P}(A) f\right](x)=\int_{a}^{b} k(x-y) f(y) d y,
$$

defined on $L^{2}(a, b)$. Here, $\mathfrak{S}=L^{2}(-\infty, \infty), A$ is the simple operator

$$
(A f)(x)=\int_{-\infty}^{\infty} k(x-y) f(y) d y,
$$

and $P$ is the projection onto functions zero outside $(a, b)$ defined by

$$
\begin{aligned}
(P f)(x) & =f(x), & & a<x<b \\
& =0, & & \text { otherwise. }
\end{aligned}
$$


When $a=0$ and $b=\infty,(1.10)$ is equivalent to the special Wiener-Hopf operator. To see this, one has only to note that by taking Fourier transforms, $A$ becomes the operator of multiplication by the Fourier transform of $k$, while $P$ becomes projection on the functions analytic in the upper half-plane and in $L^{2}$ on horizontal lines. Mapping the upper half-plane onto the unit disk conformally by means of the Cayley transformation preserves the fact that $A$ is multiplication by a complex valued function and takes $P$ into $P$. Thus, following these two transformations, $T_{P}(A)$ (with $a=0, b=\infty$ ) becomes $T_{\mathrm{P}}(\mathrm{A})$.

A very interesting result associated with the special equation (1.10) is the socalled Baxter "finite section" theorem [1]. (See also Reich [11].) This theorem says roughly that (1.10) is invertible for $b-a$ large enough if it is invertible for $a=0$, $b=\infty$. In $\S 6$, we prove an abstract version of this result, derive a set of conditions under which the result of $\S 6$ is automatically valid, and then apply this result to the finite section operator associated with (1.2) as well as to (1.10).

Finally, some remarks on terminology and notation. In the sequel, $\mathfrak{F}$ is always used to denote a Hilbert space. The scalar product and norm in $\mathfrak{S}$ are denoted by $(\cdot, \cdot)$ and $\|\cdot\|$, respectively. $A$ is always used to denote a bounded, linear operator on $\mathfrak{S} . P$ is used to denote an orthogonal projection; its range is consistently denoted by $\mathfrak{B}$. We will always take $Q=I-P$ and $\mathfrak{Q}=\mathfrak{B}^{\perp}$. To avoid trivial complications, we always assume that $\mathfrak{B}$ is neither $\{0\}$ nor $\mathfrak{F}$. It is convenient to change the usual notation and to denote the adjoint of $A$ by $A_{*}$ rather than $A^{*}$, and this is done in all that follows. The symbol $T_{P}(A)$ is always used for the operator (1.3).

$L^{2}$ is always used to denote the usual Lebesgue space with respect to Haar measure, normalized to unity. $H^{2}$ is the space defined before. Without further mention, we write $\mathrm{P}$ for orthogonal projection of $L^{2}$ onto $H^{2}$, and we write $\mathrm{A}$ for the operator defined in (1.1).

An operator $A$ is called invertible if it is one-to-one and onto $\mathfrak{S}_{\mathfrak{B}} T_{P}(A)$ is invertible if it is one-to-one and onto $\mathfrak{P}$.

2. Invertibility of $T_{P}(A)$. In this section, we derive a number of results relating to the invertibility of general Toeplitz operators. The most important of these is probably Theorem 3 below which states that $T_{P}(A)$ is invertible if and only if $A$ can be transformed in a specific way into an operator with positive real part.

We begin by defining the functional (recall $\mathfrak{Q}=\mathfrak{B}^{\perp}$ ).

$$
\rho_{P}(A)=\sup \{|(A p, q)|: p \in \mathfrak{B}, q \in \mathfrak{Q},\|A p\|=\|q\|=1\} .
$$

We can then state

TheOREM 1. Let $T_{P}(A)$ be a general Toeplitz operator. Necessary and sufficient conditions that $T_{P}(A)$ be invertible are:

(i) $A \mid \mathfrak{P}$ has a bounded inverse;

(i*) $A_{*} \mid \mathfrak{P}$ has a bounded inverse;

(ii) $\rho_{P}(A)<1$;

(ii $\left.\rho_{P}\right)\left(A_{*}\right)<1$. 
Proof. Suppose first that $T_{P}(A)$ is invertible. Then, there exists an $m>0$ such that for every $p \in \mathfrak{P}$,

$$
m\|p\| \leqq\left\|T_{P}(A) p\right\| \leqq\|A p\|,
$$

which gives the condition (i).

To derive condition (ii), note first of all that by its definition, $\rho_{P}(A) \leqq 1$. Suppose $\rho_{P}(A)=1$. Then for every $\varepsilon>0$ there exists a $p \in \mathfrak{B}$ with $\|A p\|=1$ and a $q \in \mathfrak{Q}$ with $\|q\|=1$ such that

$$
\begin{aligned}
\|Q A p\| & =\sup \left\{\left|\left(A p, q^{\prime}\right)\right|: q^{\prime} \in \mathfrak{Q},\left\|q^{\prime}\right\|=1\right\} \\
& \geqq|(A p, q)| \\
& \geqq 1-\varepsilon .
\end{aligned}
$$

Hence,

Thus, we get

$$
\begin{aligned}
1 & =\|A p\|^{2} \\
& =\|P A p\|^{2}+\|Q A p\|^{2} \\
& \geqq\|P A p\|^{2}+(1-\varepsilon)^{2} .
\end{aligned}
$$

Now,

$$
\begin{aligned}
m\|p\|^{2} & \leqq\left\|T_{P}(A) p\right\|^{2} \\
& =\|P A p\|^{2} \\
& \leqq 2 \varepsilon
\end{aligned}
$$

$$
1=\|A p\| \leqq\|A\| \cdot\|p\|
$$

which implies $\|p\| \geqq 1 /\|A\|$. Taking

$$
\varepsilon<m /\left(2\|A\|^{2}\right),
$$

we have a contradiction. This proves (ii).

It is a simple exercise to verify that $\left[T_{P}(A)\right]_{*}=T_{P}\left(A_{*}\right)$. Thus, $T_{P}(A)$ invertible implies $T_{P}\left(A_{*}\right)$ invertible, and the same reasoning as before gives $\left(\mathrm{i}_{*}\right)$ and $\left(\mathrm{ii}_{*}\right)$.

Conversely, suppose conditions (i), $\left(\mathrm{i}_{*}\right)$, (ii), and $\left(\mathrm{ii}_{*}\right)$ are satisfied. By (ii), there exists a $\delta>0$ such that for every $p \in \mathfrak{P}$ we have

Consequently,

$$
\begin{aligned}
\|Q A p\| & =\sup \{|(A p, q)|: q \in \mathbb{Q},\|q\|=1\} \\
& \leqq(1-\delta)\|A p\| .
\end{aligned}
$$

$$
\begin{aligned}
(1-\delta)\|A p\| & \geqq\|Q A p\| \\
& =\|(I-P) A p\| \\
& \geqq\|A p\|-\|P A p\|,
\end{aligned}
$$

or $\left\|T_{P}(A) p\right\| \geqq \delta\|A p\|$. By (i), there is a constant $m>0$ such that $\|A p\| \geqq m\|p\|$ for every $p \in \mathfrak{B}$. Therefore,

$$
\left\|T_{P}(A) p\right\| \geqq m \delta\|p\|,
$$

and $T_{P}(A)$ is one-to-one and has a closed range. 
We proved (2.1) using (i) and (ii). In the same way, using ( $\left.\mathrm{i}_{*}\right)$ and $\left(\mathrm{ii}_{*}\right)$, we can show that $T_{P}\left(A_{*}\right)$ is bounded below, so that $T_{P}\left(A_{*}\right)$ is also one-to-one. Since the range of $T_{P}(A)$ is the orthogonal complement of the nullspace of $\left[T_{P}(A)\right]_{*}=T_{P}\left(A_{*}\right)$, it follows that the range of $T_{P}(A)$ is all of $\mathfrak{B}$. Thus, $T_{P}(A)$ is invertible.

We need Theorem 1 in the particularly simple form it takes when $A$ is unitary. We state this form of the theorem as

COROLlaRY 1. Let $U$ be a unitary operator on $\mathfrak{K}$. Necessary and sufficient conditions that $T_{P}(U)$ be invertible are:

(iii) $\|Q U P\|<1$;

(iv) $\|P U Q\|<1$.

Consequently, $T_{P}(U)$ is invertible if and only if $T_{Q}(U)$ is invertible.

Proof. We apply Theorem 1 with $A=U$. Conditions (i) and (i $\mathrm{i}_{*}$ ) are automatic since $U$ is unitary. Also, and again since $U$ is unitary, $\rho_{P}(U)=\|Q U P\|$, so that (ii) is equivalent to (iii). Next, $\rho_{P}\left(U_{*}\right)=\left\|Q U_{*} P\right\|=\left\|\left(Q U_{*} P\right)_{*}\right\|=\|P U Q\|$, so that (ii $\left.{ }_{*}\right)$ is equivalent to (iv). This proves the first part of the corollary.

To prove the second part, we note that conditions (iii) and (iv) are symmetric in $P$ and $Q$, so that the invertibility of $T_{P}(U)$ and $T_{Q}(U)$ must be equivalent.

A less immediate result on the invertibility of Toeplitz operators associated with a unitary operator is (see [9] and [10])

THEOREM 2. Let $U$ be a unitary operator on $\mathfrak{g}$. The operator $T_{P}(U)$ is invertible if and only if there exists a bounded, invertible operator $G$ on $\mathfrak{S}$ that takes $\mathfrak{B}$ onto itself and such that

$$
\|U-G\|<1 .
$$

Proof. Suppose $T_{P}(U)$ is invertible. Set

$$
G=P U P+Q U Q .
$$

By the last sentence of Corollary 1, $G$ may be considered to be the direct sum of two invertible operators and so is itself invertible. Moreover, Corollary 1 shows that for some $\delta>0$

$$
\|Q U P\| \leqq 1-\delta, \quad\|P U Q\| \leqq 1-\delta .
$$

$G$ clearly takes $\mathfrak{P}$ and $\mathfrak{Q}$ onto themselves. Thus, if $p \in \mathfrak{P}$ and $q \in \mathfrak{Q}$, we have

$$
\begin{aligned}
((U-G) p,(U-G) q) & =-(U p, G q)-(G p, U q) \\
& =-(U p, Q U q)-(P U p, U q) \\
& =-(Q U p, U q)-(P U p, U q) \\
& =-(U p, U q) \\
& =-(p, q) \\
& =0 .
\end{aligned}
$$


Suppose $f \in \mathfrak{H},\|f\|=1$. Write $f=p+q$, where $p \in \mathfrak{B}, q \in \mathfrak{Q}$. (2.2) shows that $(U-G) p$ is orthogonal to $(U-G) q$. Therefore,

$$
\begin{aligned}
\|(U-G) f\|^{2} & =\|(U-G) p+(U-G) q\|^{2} \\
& =\|(U-G) p\|^{2}+\|(U-G) q\|^{2} \\
& =\|Q U p\|^{2}+\|P U q\|^{2} \\
& \leqq(1-\delta)^{2}\left(\|p\|^{2}+\|q\|^{2}\right) \\
& =(1-\delta)^{2}\|f\|^{2} .
\end{aligned}
$$

This means that $\|U-G\|<1$.

Conversely, suppose $G$ is an invertible operator taking $\mathfrak{P}$ onto itself and such that $\|U-G\|<1$. Since $U$ is unitary, we have

$$
\begin{aligned}
\left\|T_{P}(I)-T_{P}\left(U_{*} G\right)\right\| & \leqq\left\|I-U_{*} G\right\| \\
& =\|U-G\| \\
& <1 .
\end{aligned}
$$

Since $T_{P}(I)$ is just the identity on $\mathfrak{P}$, it follows that $T_{P}\left(U_{*} G\right)$ is invertible. Since $G$ takes $\mathfrak{B}$ onto itself,

$$
T_{P}\left(U_{*} G\right)=T_{P}\left(U_{*}\right) T_{P}(G) .
$$

Since $T_{P}(G)$ is invertible, it follows that $T_{P}\left(U_{*}\right)$ is invertible. So, then, is $T_{P}(U)$.

It should be noted that, according to the construction of $G$ in the above proof, not only does $G$ have $\mathfrak{P}$ as an invariant subspace, but both $\mathfrak{P}$ and $\mathfrak{Q}$ reduce $G$.

The following proposition was obtained by Pousson [9] and Rabindranathan [10] for $H^{2}$ spaces. The proof we give follows that of Pousson.

Lemma 1. Let $A$ be invertible. Then, it can be factored in the form $A=U B$, where $U$ is unitary and $B$ is an invertible operator taking $\mathfrak{B}$ onto itself. $T_{P}(A)$ is invertible if and only if the corresponding operator $T_{P}(U)$ is invertible.

Proof. $A_{*} A$ is a bounded, positive operator with a bounded universe. Hence, by a theorem of Shinbrot [12], we can factor $A_{*} A$ in the form

$$
A_{*} A=B_{*} B,
$$

where $B$ is invertible and takes $\mathfrak{B}$ onto itself and $B_{*}$ takes $\mathscr{Q}$ onto itself. Thus, we have $A=U B$, where $U=A_{*}^{-1} B_{*}$. We have

$$
\begin{aligned}
U U_{*} & =A_{*}^{-1} B_{*} B A^{-1} \\
& =A_{*}^{-1} A_{*} A A^{-1} \\
& =I .
\end{aligned}
$$

A similar calculation shows that $U_{*} U=I$. Therefore, $U$ is unitary.

To prove the last sentence of the lemma, we notice that since $\mathfrak{P}$ is an invariant subspace of $B$,

while $T_{P}(B)$ is invertible.

$$
T_{P}(A)=T_{P}(U) T_{P}(B)
$$

Notice that Lemma 1 gives the following abstract version of a theorem of Beurling [2] about factorization into inner and outer factors. 
Corollary 2. Let $A$ be invertible and map $\mathfrak{P}$ into itself. Then $A=U B$, where $U$ is unitary and takes $\mathfrak{P}$ into itself, while $B$ is invertible and takes $\mathfrak{P}$ onto itself.

Corollary 2 is not the complete abstract version of Beurling's theorem because of the hypothesis we make that $A$ is bounded. It is possible to extend the corollary to include the operator (1.1) with $a(\theta) \in H^{2}$ (which is the hypothesis of Beurling's theorem) but we do not do so here since this would take us out of our basic framework of bounded operators.

There are a number of possible proofs of the next result; we give one that depends on Lemma 1.

Corollary 3. Suppose $A$ is invertible. Then $T_{P}(A)$ is invertible if and only if $T_{Q}\left(A^{-1}\right)$ is invertible. Further,

$$
\left\|\left[T_{Q}\left(A^{-1}\right)\right]^{-1}\right\| \leqq 2\|A\|^{3}\left\|\left[T_{P}(A)\right]^{-1}\right\|^{2} .
$$

Proof. We may write $A=U B$, where $B$ is invertible and takes $\mathfrak{P}$ onto itself. As a consequence (or, by the result in [12]), $B_{*}$ takes $\mathscr{D}$ onto itself. Since $T_{P}(A)$ is invertible, so is $T_{P}(U)$.

Now write $A_{*}^{-1}=U B_{*}^{-1}$. Then

$$
T_{Q}\left(A_{*}^{-1}\right)=T_{Q}(U) T_{Q}\left(B_{*}^{-1}\right) .
$$

Since $U$ is unitary and $T_{P}(U)$ is invertible, Corollary 1 shows that $T_{Q}(U)$ is invertible. Hence, $T_{Q}\left(A_{*}^{-1}\right)$ and $\left[T_{Q}\left(A_{*}^{-1}\right)\right]_{*}=T_{Q}\left(A^{-1}\right)$ are invertible. By interchanging the roles of $P$ and $Q$ and of $A$ and $A^{-1}$, we get the converse statement.

Suppose now that $\left\|\left[T_{P}(A)\right]^{-1}\right\| \leqq m$. Using the decomposition $A=U B$, we obtain for every $p \in \mathfrak{B}$,

$$
\begin{aligned}
\left\|T_{P}(U) B p\right\| & =\left\|T_{P}(A) p\right\| \\
& \geqq \frac{1}{m}\left\|B^{-1} B p\right\| \\
& \geqq \frac{1}{m\|A\|}\|B p\| .
\end{aligned}
$$

Since $B p$ varies over all of $\mathfrak{B}$ as $p$ varies over $\mathfrak{B},(2.3)$ gives a bound for $\left\|\left[T_{P}(U)\right]^{-1}\right\|$.

By Theorem $1, \rho_{P}(U)=1-\delta$ where $\delta>0$. Thus it follows that for every $\varepsilon>0$ there exists a $p \in \mathfrak{P}$ with $\|p\|=1$ such that $\|Q U p\| \geqq 1-\delta-\varepsilon$. Consequently,

$$
\begin{aligned}
1 & =\|U p\|^{2} \\
& =\|P U p\|^{2}+\|Q U p\|^{2} \\
& \geqq\|P U p\|^{2}+(1-\delta-\varepsilon)^{2} .
\end{aligned}
$$

Thus,

$$
1 /\left(m^{2}\|A\|^{2}\right) \leqq\|P U p\|^{2} \leqq 2(\delta+\varepsilon),
$$

from which we get $\delta \geqq 1 /\left(2 m^{2}\|A\|^{2}\right)$. By the proof of Theorem 1, on the other hand,

$$
\left\|\left[T_{Q}(U)\right]^{-1}\right\| \leqq 1 / \delta \leqq 2 m^{2}\|A\|^{2} .
$$


Now use the decomposition $A_{*}^{-1}=U B_{*}^{-1}$. We get

But

$$
\begin{aligned}
\left\|\left[T_{Q}\left(A^{-1}\right)\right]^{-1}\right\| & =\left\|\left[T_{Q}\left(A_{*}^{-1}\right)\right]^{-1}\right\| \\
& \leqq\left\|\left[T_{Q}\left(B_{*}^{-1}\right)\right]^{-1}\right\| \cdot\left\|\left[T_{Q}(U)\right]^{-1}\right\| .
\end{aligned}
$$

$$
\left\|\left[T_{Q}\left(B_{*}^{-1}\right)\right]^{-1}\right\|=\left\|B_{*}\right\|=\|A\|,
$$

and this gives the final result.

We shall say that an operator $A$ has strongly positive real part if there exists a $\delta>0$ such that

for all $f \in \mathfrak{S}$.

$$
\operatorname{Re}(A f, f) \geqq \delta\|f\|^{2}
$$

LEMMA 2. If $A$ has strongly positive real part, then $T_{P}(A)$ is invertible.

Proof. Let $p \in \mathfrak{B},\|p\|=1$. Then

$$
\begin{aligned}
\left\|T_{P}(A) p\right\| & \geqq\left|\left(T_{P}(A) p, p\right)\right| \\
& =|(A p, p)| \\
& \geqq \delta
\end{aligned}
$$

by hypothesis. Similarly,

$$
\begin{aligned}
\left\|T_{P}\left(A_{*}\right) p\right\| & \geqq\left|\left(A_{*} p, p\right)\right| \\
& \geqq \delta .
\end{aligned}
$$

The inequalities (2.4) and (2.5) are equivalent to the invertibility of $T_{P}(A)$.

THEOREM 3. Let $A$ be invertible. Then, $T_{P}(A)$ is invertible if and only if there exists a bounded, invertible operator $H$ taking $\mathfrak{B}$ onto itself such that $A H$ has strongly positive real part.

Proof. $A$ is invertible. Therefore, $A_{*}$ is invertible. Applying Lemma 1 to $A_{*}$, we find that $A_{*}=U B$, where $U$ is unitary and $B$ is an invertible operator taking $\mathfrak{B}$ onto itself. Let $T_{P}(A)$ be invertible. By Lemma 1 (applied to $T_{P}\left(A_{*}\right)$ ), $T_{P}(U)$ is invertible, and so by Theorem 2 there exists a bounded, invertible operator $G$ on $\mathfrak{S}$ taking $\mathfrak{P}$ onto itself and such that

Consequently,

$$
\|U-G\|=1-\eta, \quad \eta>0 .
$$

$$
((U-G) f,(U-G) f) \leqq(1-\eta)\|f\|^{2}
$$

for every $f \in \mathfrak{S}$. Expanding, we find that

$$
\left(\left(G_{*} U+U_{*} G\right) f, f\right) \geqq \eta\|f\|^{2}
$$

or, in other words, $G_{*} U+U_{*} G \geqq \eta I$. From this, we get

$$
B_{*}\left(G_{*} U+U_{*} G\right) B \geqq \eta B_{*} B=\eta A A_{*},
$$

the last equality following from the construction in the proof of Lemma 1. 
Let $H=G B$. Then, we have

$$
H_{*} U B+B_{*} U_{*} H \geqq \eta A A_{*} .
$$

Since $A A_{*}$ is strongly positive, this inequality shows that $B_{*} U_{*} H=A H$ has strongly positive real part. Further, $H$ is invertible and takes $\mathfrak{P}$ onto itself, since both $G$ and $B$ have these properties. This proves the necessity.

Conversely, suppose there exists an operator $H$ satisfying the conditions of the theorem. By Lemma 2, $T_{P}(A H)$ is then invertible. But $T_{P}(A H)=T_{P}(A) T_{P}(H)$, and since $T_{P}(H)$ is invertible, so is $T_{P}(A)$.

Corollary 4. Let $A$ be invertible. Let $H$ be the operator constructed in Theorem 3. The constant $\delta$ occurring in the inequality $\operatorname{Re}(A H f, f) \geqq \delta\|f\|^{2}$ can be taken to be

$$
\delta=\frac{1}{2\|A\|^{2}\left\|A^{-1}\right\|^{2}\left\|\left[T_{P}(A)\right]^{-1}\right\|^{2}} .
$$

Proof. If we write $A=U B$, then Theorem 1 shows that $\rho_{P}(U)=1-\eta, \eta>0$. The proof of Corollary 3 shows that we may take

$$
\eta=\frac{1}{2\|A\|^{2}\left\|\left[T_{P}(A)\right]^{-1}\right\|^{2}}
$$

From the proof of Theorem 3, the constant $\delta$ can be chosen to be $\eta /\left\|A^{-1}\right\|^{2}$. The corollary follows.

3. Invertibility of $A$. Our main purpose in this paper is to discuss invertibility conditions for $T_{P}(A)$ when $A$ is invertible. But we shall digress here to discuss the related question of the invertibility of $A$ given that $T_{P}(A)$ is invertible. Generally, it is not true that if $T_{P}(A)$ is invertible then $A$ itself is invertible. For example, we may take $A$ to be the identity operator on $\mathfrak{P}$ and the zero operator on $\mathfrak{Q}$. This operator $A$ is clearly not invertible, but $T_{P}(A)$ is just the identity on $\mathfrak{P}$ and so is invertible. In the case of the special Wiener-Hopf operator $T_{\mathrm{P}}(\mathrm{A})$, however, it is known that the invertibility of $T_{\mathrm{P}}(\mathrm{A})$ implies that of $\mathrm{A}$ [3] and [6]. This comes about, of course, because of the special nature of the operator A. However, an appropriate abstract version of this fact also exists; it has as an immediate consequence the result just described for the special Wiener-Hopf operator.

THEOREM 4. Let $\mathscr{S}$ be a collection of operators on $\mathfrak{S}$ with the following properties:

(v) there exist two positive numbers $m$ and $M$ such that for every $S \in \mathscr{S}$ and for every $f \in \mathfrak{S}$,

$$
m\|f\| \leqq\|S f\| \leqq M\|f\|
$$

(vi) every element of $\mathscr{S}$ commutes with $A$ and with $A_{*}$;

(vii) the set $\{S p: S \in \mathscr{S}, p \in \mathfrak{B}\}$ is dense in $\mathfrak{S}$.

Then $A$ is invertible if $T_{P}(A)$ is invertible.

If, in addition to (v), (vi), and (vii), we have

(viii) all elements of $\mathscr{S}$ are unitary operators, then,

$$
\left\|T_{P}(A)\right\|=\|A\| \text {. }
$$


Proof. If $S \in \mathscr{S}$, let $S^{-1}$ denote the left inverse of $S$. Then we have

$$
(1 / M)\|f\| \leqq\left\|S^{-1} f\right\| \leqq(1 / m)\|f\|,
$$

for every $f$ in the domain of $S^{-1}$. From Theorem 1 we get the existence of a $\delta>0$ such that for every $p \in \mathfrak{P},\|A p\| \geqq \delta\|p\|$. Thus we have, for every $S \in \mathscr{S}$ and every $p \in \mathfrak{P}$,

$$
\begin{aligned}
(1 / m)\|A S p\| & =(1 / m)\|S A p\| \\
& \geqq\left\|S^{-1} S A p\right\| \\
& \geqq \delta\left\|S^{-1} S p\right\| \\
& \geqq(\delta / M)\|S p\| .
\end{aligned}
$$

From this inequality and the hypothesis (vii) it follows that $A$ is one-to-one and has a closed range. The same argument applied to $A_{*}$ shows that $A_{*}$ is one-to-one, so that $A$ is onto. Therefore, $A$ is invertible.

Now, suppose that (viii) holds. Then

$$
\begin{aligned}
\left\|T_{P}(A)\right\| & =\sup \left\{\left|\left(T_{P}(A) p, p^{\prime}\right)\right|: p, p^{\prime} \in \mathfrak{P},\|p\|=\left\|p^{\prime}\right\|=1\right\} \\
& =\sup \left\{\left|\left(A S p, S p^{\prime}\right)\right|: S \in \mathscr{S}, p, p^{\prime} \in \mathfrak{B},\|p\|=\left\|p^{\prime}\right\|=1\right\} \\
& =\|A\| .
\end{aligned}
$$

This completes the proof of Theorem 4.

We denote the spectrum of $A$ by $\sigma(A)$. Then we have

COROLlaRy 5. If there exists a family $\mathscr{S}$ with the properties (v), (vi), and (vii), then

$$
\sigma(A) \subset \sigma\left(T_{P}(A)\right) .
$$

Corollary 5 follows by applying Theorem 4 to the operator $\lambda I-A$ instead of $A$ with $\lambda$ in the resolvent set of $T_{P}(A)$.

Corollary 6. We have $\sigma(\mathrm{A}) \subset \sigma\left(T_{\mathrm{P}}(\mathrm{A})\right)$. Also, $\|\mathrm{A}\|=\left\|T_{\mathrm{P}}(\mathrm{A})\right\|$.

Corollary 6 follows from Theorem 4 and Corollary 5 by taking $\mathscr{S}$ to be the group of operators on $L^{2}$ consisting of multiplication by characters of the circle group. That conditions (v), (vi), (vii), and (viii) hold is immediate.

The first part of the proof of Theorem 4 is in essence due to Hartman-Wintner [6]. The first statement of Corollary 6 is usually called the Hartman-Wintner spectral inclusion theorem [6]. The second statement of Corollary 6 appears in [18].

4. Invertibility and factorization. The purpose of this section is to show that if $A$ is an invertible operator, then $T_{P}(A)$ is invertible if and only if $A$ can be factored in a certain canonical way, which in the classical theory is very familiar. Before we state and prove the factorization theorem, it will be convenient to prove a simple lemma.

LEMMA 3. $T_{P}(A)$ is invertible if and only if $A \mid \mathfrak{P}$ has a bounded inverse, and $A \mathfrak{P}+\mathfrak{Q}=\mathfrak{S}$, where $\dot{+}$ indicates a direct sum. 
Proof. Suppose $T_{P}(A)$ is invertible. Then we know from Theorem 1 that $A \mid \mathfrak{P}$ has a bounded inverse. Now suppose

$$
A p+q=0, \quad p \in \mathfrak{P}, q \in \mathfrak{Q} .
$$

Then, $T_{P}(A) p=0$, which implies $p=0$, which in turn implies $q=0$. This shows the sum is direct. Next, given any $f \in \mathfrak{H}$, there exists a $p \in \mathfrak{B}$ such that $T_{P}(A) p=P f$. This means there exists a $q \in \mathbb{D}$, such that $A p+q=f$. This proves the first half of the lemma.

For the converse, suppose the conditions of the lemma are satisfied. Then, $P A \mathfrak{P}=P \mathfrak{F}$, so that $T_{P}(A)$ is onto. If $T_{P}(A) p=0$, then $A p \in \mathfrak{Q}$. But the sum of $A \mathfrak{P}$ and $\mathfrak{Q}$ being direct then implies $A p=0$, so that $p=0$, and $T_{P}(A)$ is one-to-one. This completes the proof of the lemma.

It should be noted that Lemma 3 has as a consequence another proof of the fact already proved in Corollary 3 that $T_{P}(A)$ is invertible if and only if $T_{Q}\left(A^{-1}\right)$ is invertible.

We now turn to the main result of this section.

Theorem 5. Let $A$ be invertible. Then, $T_{P}(A)$ is invertible if and only if $A$ can be factored in the form $A=A_{-} A_{+}$, where $A_{-}$and $A_{+}$are bounded, invertible operators, $A_{-}$takes $\mathfrak{Q}$ onto itself, and $A_{+}$takes $\mathfrak{B}$ onto itself.

Moreover, if $T_{P}(A)$ is invertible, then

$$
\left[T_{P}(A)\right]^{-1}=A_{+}^{-1} P A_{-}^{-1} \mid \mathfrak{P} .
$$

Proof. We suppose first that $A$ can be factored and prove that $T_{P}(A)$ is invertible. Since $A_{-}$is invertible and takes $\mathfrak{Q}$ onto itself, it follows that $A_{-}^{-1}$ takes $\mathfrak{Q}$ onto itself. Thus, $T_{Q}\left(A_{-}^{-1}\right)$ is invertible. By Corollary 3 , then, $T_{P}\left(A_{-}\right)$is invertible. Since $T_{P}\left(A_{+}\right)$is invertible and $T_{P}(A)=T_{P}\left(A_{-}\right) T_{P}\left(A_{+}\right)$, it follows that $T_{P}(A)$ is invertible.

Next, we prove (4.1). Let $p \in \mathfrak{B}$, and define $f=A_{+}^{-1} P A_{-}^{-1} p$. Since $A_{+}$is invertible and takes $\mathfrak{P}$ onto itself, $A_{+}^{-1}$ also takes $\mathfrak{P}$ onto itself, and $f \in \mathfrak{B}$. Therefore,

$$
\begin{aligned}
T_{P}(A) f & =P A f \\
& =P A_{-} P A_{-}^{-1} p \\
& =P A_{-}(I-Q) A_{-}^{-1} p \\
& =p
\end{aligned}
$$

since $A_{-}$takes $\mathfrak{Q}$ into itself. This proves (4.1).

The hard part of Theorem 5 is the necessity. We note first that there is no loss in generality in assuming that $A$ has strongly positive real part. For, if $T_{P}(A)$ is invertible, Theorem 3 tells us that there is an invertible operator $H$ that takes $\mathfrak{P}$ onto itself and such that $A H$ has strongly positive real part. Thus, if we can prove Theorem 5 for such operators, we shall have a factorization of the form $A H=B_{-} B_{+}$, $B_{-}$and $B_{+}$having the properties specified for $A_{-}$and $A_{+}$, respectively. Taking $A_{-}=B_{-}$and $A_{+}=B_{+} H^{-1}$, we then have a factorization of $A$ with all the required properties. We assume therefore that $A$ has strongly positive real part. 
When this is the case, the spectrum of $A$ is a compact set in the open right half of the complex plane. Let $\gamma$ be any Jordan curve in the right-half plane containing the spectrum of $A$ in its interior. Define two operators by the formulas

$$
A^{ \pm 1 / 2}=\frac{1}{2 \pi i} \int_{\gamma} \lambda^{ \pm 1 / 2}(\lambda I-A)^{-1} d \lambda,
$$

where, for the sake of being definite, we specify the branch of $\lambda^{1 / 2}$ so that it is positive when $\lambda$ is a positive real number. The operators $A^{ \pm 1 / 2}$ are bounded, $A=\left[A^{1 / 2}\right]^{2}$ and $A^{-1 / 2}$ is the inverse of $A^{1 / 2}$.

Let $\pi$ be a set with the same cardinality as the dimension of $\mathfrak{P}$, and let $\chi$ be a set disjoint from $\pi$ with the same cardinality as the dimension of $\mathfrak{Q}$. We denote by $\left\{\varphi_{n}: n \in \pi\right\}$ and by $\left\{\varphi_{n}: n \in \chi\right\}$ orthonormal bases for $\mathfrak{B}$ and for $\mathfrak{Q}$, respectively. Similarly, we denote by $\left\{\psi_{n}: n \in \pi\right\}$ and by $\left\{\psi_{n}: n \in \chi\right\}$ orthonormal bases for the spaces $A_{*}^{1 / 2} \mathfrak{B}$ and $A_{*}^{-1 / 2} \mathfrak{Q}$, respectively. Define a linear operator $V$ in $\mathfrak{H}$ by means of the formula

$$
V f=\sum_{\pi \cup x}\left(f, \psi_{n}\right) \varphi_{n}
$$

wherever it makes sense. We shall show that $V$ is a bounded, invertible operator defined on all of $\mathfrak{S}$.

To show that $V$ is defined on all of $\mathfrak{S}$, we notice that since $\mathfrak{P}$ is orthogonal to $\mathfrak{Q}$, the set $\left\{\varphi_{n}: n \in \pi \cup \chi\right\}$ is orthonormal. Therefore,

$$
\begin{aligned}
\|V f\|^{2} & =\sum_{\pi \cup x}\left|\left(f, \psi_{n}\right)\right|^{2} \\
& =\sum_{\pi}\left|\left(f, \psi_{n}\right)\right|^{2}+\sum_{x}\left|\left(f, \psi_{n}\right)\right|^{2} \\
& \leqq 2\|f\|^{2},
\end{aligned}
$$

by Bessel's inequality, since the sets $\left\{\psi_{n}: n \in \pi\right\}$ and $\left\{\psi_{n}: n \in \chi\right\}$ are separately orthonormal. (4.3) also shows that $V$ is bounded.

There are several ways to prove that $V$ is invertible. Perhaps the easiest way is to prove that $V_{*}$ is invertible. We obtain first a formula for $V_{*}$. For every $f$ and $g$ in $\mathfrak{S}$, we have

$$
(V f, g)=\sum_{\pi \cup x}\left(f, \psi_{n}\right)\left(\varphi_{n}, g\right)
$$

Since $\left\{\varphi_{n}: n \in \pi \cup \chi\right\}$ is orthonormal,

$$
\sum_{\pi \cup x}\left|\left(g, \varphi_{n}\right)\right|^{2}<\infty
$$

and from this it follows that, since $\left\{\psi_{n}: n \in \pi\right\}$ and $\left\{\psi_{n}: n \in \chi\right\}$ are orthonormal, the sums

$$
r=\sum_{n \in \pi}\left(g, \varphi_{n}\right) \psi_{n}
$$


and

converge in $\mathfrak{S}$. It follows that

$$
s=\sum_{n \in \chi}\left(g, \varphi_{n}\right) \psi_{n}
$$

$$
\sum_{\pi \cup x}\left(g, \varphi_{n}\right) \psi_{n}
$$

converges in $\mathfrak{S}$ to $r+s$. Thus, we have

$$
(V f, g)=\left(f, \sum_{\pi \cup x}\left(g, \varphi_{n}\right) \psi_{n}\right)=\left(f, V_{*} g\right),
$$

and from this we get

$$
V_{*} g=\sum_{\pi \cup x}\left(g, \varphi_{n}\right) \psi_{n}
$$

It is clear from this formula that the range of $V_{*}$ is the direct sum $A_{*}^{1 / 2} \mathfrak{P}+A_{*}^{-1 / 2} \mathfrak{Q}$. The fact that this sum is direct follows from Lemma 3 . Indeed, since $T_{P}\left(A_{*}\right)$ is invertible, $A_{*}^{1 / 2} p+A_{*}^{-1 / 2} q=0$ with $p \in \mathfrak{B}$ and $q \in \mathbb{Q}$ implies $A_{*} p+q=0$, and Lemma 3 shows that this means $p=q=0$. The same proof also shows that $V_{*}$ is one-to-one.

Moreover, since $A_{*} \mathfrak{P} \mathfrak{+}=\mathfrak{H}_{\mathcal{E}}$, it follows that $A_{*}^{1 / 2} \mathfrak{P}+A_{*}^{-1 / 2} \mathfrak{Q}=\mathfrak{F}$, so that the range of $V_{*}$ is all of $\mathfrak{B}$, and $V_{*}$ is invertible.

We now define

$$
\begin{aligned}
& A_{+}=V A^{1 / 2}, \\
& A_{-}=A^{1 / 2} V^{-1} .
\end{aligned}
$$

Clearly, $A_{+}$and $A_{-}$are bounded, invertible operators satisfying $A_{-} A_{+}=A$. To complete the proof of Theorem 5 , it remains to show that

$$
\begin{aligned}
& A_{+} \mathfrak{P}=\mathfrak{P}, \\
& A_{-} \mathfrak{Q}=\mathfrak{Q} .
\end{aligned}
$$

First of all, we notice that since $A^{1 / 2} \mathfrak{B}$ and $A_{*}^{-1 / 2} \mathfrak{Q}$ are orthogonal, it follows from the definition of $V$ that $A_{+} \mathfrak{B} \subseteq \mathfrak{P}$. Similarly, since $A^{-1 / 2} \mathfrak{Q}$ and $A_{*}^{1 / 2} \mathfrak{B}$ are orthogonal, $A_{-}^{-1} \mathfrak{Q} \subseteq \mathfrak{Q}$. Now Lemma 3 implies

$$
A^{1 / 2} \mathfrak{P} \dot{+} A^{-1 / 2} \mathfrak{Q}=\mathfrak{H} .
$$

Since the range of $V$ is $\mathfrak{H}$, this means that

$$
A_{+} \mathfrak{P}+A_{-}^{-1} \mathfrak{Q}=V\left(A^{1 / 2} \mathfrak{P}+A^{-1 / 2} \mathfrak{Q}\right)=\mathfrak{H} .
$$

Consequently, we must have $A_{+} \mathfrak{B}=\mathfrak{P}$ and $A_{-}^{-1} \mathfrak{Q}=\mathfrak{Q}$. Since $A_{-}$is invertible, it follows that $A_{-} \mathfrak{Q}=\mathfrak{Q}$. The proof of Theorem 5 is complete.

Let $P_{1}$ and $P_{2}$ be two, possibly different projections onto subspaces $\mathfrak{B}_{1}$ and $\mathfrak{B}_{2}$ of $\mathfrak{S}_{\mathrm{C}}$. An interesting generalization of the general Toeplitz operator is $T_{12}(A)=$ $P_{1} A \mid \mathfrak{P}_{2}$. We say that $T_{12}(A)$ is invertible if it is one-to-one and maps $\mathfrak{P}_{2}$ onto $\mathfrak{P}_{1}$. 
Let $Q_{1}=I-P_{1}, Q_{2}=I-P_{2}, \mathfrak{Q}_{1}=\mathfrak{B}_{1}^{\frac{1}{1}}, \mathfrak{Q}_{2}=\mathfrak{P}_{2}^{\frac{1}{2}}$. The following corollary of Theorem 5 has important consequences for unbounded Wiener-Hopf operators.

COROLlaRY 7. Let

$$
\operatorname{dim} \mathfrak{P}_{1}=\operatorname{dim} \mathfrak{P}_{2}, \quad \operatorname{dim} \mathfrak{Q}_{1}=\operatorname{dim} \mathfrak{Q}_{2} .
$$

Then, $T_{12}(A)$ is invertible if and only if $A$ can be factored in either of the forms

$$
\begin{aligned}
A & =B_{-} B_{+} \\
& =C_{-} C_{+},
\end{aligned}
$$

where $B_{-}, B_{+}, C_{-}, C_{+}$are invertible operators on $\mathfrak{S}_{2}, B_{-}$maps $\mathfrak{Q}_{1}$ onto $\mathfrak{Q}_{2}, B_{+}$ maps $\mathfrak{P}_{2}$ onto itself, $C_{-}$maps $\mathfrak{Q}_{1}$ onto itself, and $C_{+}$maps $\mathfrak{P}_{2}$ onto $\mathfrak{P}_{1}$.

If $T_{12}(A)$ is invertible, we have

$$
\begin{aligned}
{\left[T_{12}(A)\right]^{-1} p } & ={ }^{\prime} B_{+}^{-1} P_{2} B_{-}^{-1} p \\
& =C_{+}^{-1} P_{1} C_{-}^{-1} p .
\end{aligned}
$$

Proof. We prove (4.6) and (4.8). The proofs of (4.7) and (4.9) are similar. Under the hypothesis (4.5), there is a unitary operator $W$ such that $P_{1}=W_{*} P_{2} W$. Therefore, $T_{12}(A)$ is invertible if and only if $T_{P_{2}}(W A)$ is invertible. According to Theorem 5, $T_{P_{2}}(W A)$ is invertible if and only if $W A=A_{-} A_{+}, A_{-}$and $A_{+}$are invertible, $A_{-}$ maps $\mathfrak{Q}_{2}$ onto itself, and $A_{+}$maps $\mathfrak{P}_{2}$ onto itself. Writing

$$
B_{-}=W_{*} A_{-}, \quad B_{+}=A_{+},
$$

we see that (4.6) is equivalent to the invertibility of $T_{12}(A)$. (4.8) follows from (4.1).

The factorization of Theorem 5 is certainly not unique. It is of some interest, therefore, to see how much can be said of each of the factors. This is the content of the next result.

THEOREM 6. If $A$ satisfies the hypotheses of Theorem 5 , and if

$$
\begin{aligned}
A & =A_{-} A_{+} \\
& =B_{-} B_{+}
\end{aligned}
$$

are two decompositions of $A$ satisfying the conditions of that theorem, then there exists a bounded, invertible operator $C$ that is reduced by $\mathfrak{\Re}$, satisfying

$$
B_{+}=C A_{+}, \quad B_{-}=A_{-} C^{-1} .
$$

If $A \geqq 0$, while $A_{-}=\left(A_{+}\right)_{*}$ and $B_{-}=\left(B_{+}\right)_{*}$, then $C$ is unitary.

Proof. We have $A_{-} A_{+}=B_{-} B_{+}$, so that $B_{-}^{-1} A_{-}=B_{+} A_{+}^{-1}$. Consequently,

$$
B_{-}^{-1} A_{-} \mathfrak{Q}=B_{+} A_{+}^{-1} \mathfrak{Q}=\mathfrak{Q} .
$$

But since $B_{+} A_{+}^{-1}$ is an invertible operator taking $\mathfrak{B}$ onto itself, it follows that $\left(B_{+} A_{+}^{-1}\right)_{*} \mathfrak{Q}=\mathfrak{Q}$. Thus, taking $C=B_{+} A_{+}^{-1}=B_{-}^{-1} A_{-}$, we obtain the first part of the theorem. 
The proof of Theorem 5 shows that if $A \geqq 0$, then $A_{-}$can be taken as $\left(A_{+}\right)_{*}$. In that case, we have

$$
\begin{aligned}
C_{*} C & =\left(A_{+}^{-1}\right)_{*}\left(B_{+}\right)_{*} B_{+} A_{+}^{-1} \\
& =A_{-}^{-1} B_{-} B_{+} A_{+}^{-1} \\
& =A_{-}^{-1} A A_{+}^{-1} \\
& =I
\end{aligned}
$$

and similarly $C C_{*}=I$, which shows that $C$ is unitary.

5. An inversion formula. The method of proof of Theorem 5 is constructive and therefore can be used to find an explicit inversion formula for the inverse of $T_{P}(A)$. When $A \geqq 0$, an analogous formula was derived in [12]. This formula proved its worth in [13], where it was used to discuss unbounded positive Wiener-Hopf operators, and in [14], where it was used to solve certain difficult integral equations.

In this section, we shall always assume the dimension of $\mathfrak{H}$ to be countable. This has as a consequence the fact that an orthonormal basis for any subspace of $\mathfrak{H}$ can be constructed explicitly by means of the Gram-Schmidt process. To avoid certain notational difficulties, we shall also assume that $\mathfrak{B}$ and $\mathfrak{Q}$ are infinite dimensional. If this is not the case, certain minor modifications in the following formulas will have to be made.

To begin, we assume that $A$ has strongly positive real part. We can then define square roots by (4.2); we assume that $A_{*}^{1 / 2}$ and $A_{*}^{-1 / 2}$ can be found explicitly. In that case, orthonormal bases $\left\{\psi_{n}\right\}_{1}^{\infty}$ and $\left\{\psi_{n}\right\}_{-\infty}^{-1}$ for $A_{*}^{1 / 2} \mathfrak{P}$ and $A^{-1 / 2} \mathfrak{Q}$, respectively, can be constructed by choosing sequences $\left\{\omega_{n}\right\}_{1}^{\infty}$ and $\left\{\omega_{n}\right\}_{-\infty}^{-1}$ that are bases in $\mathfrak{P}$ and in $\mathfrak{Q}$ and orthonormalizing $\left\{A_{*}^{1 / 2} \omega_{n}\right\}_{1}^{\infty}$ and $\left\{A_{*}^{-1 / 2} \omega_{n}\right\}_{-\infty}^{-1}$. We denote by $\left\{\varphi_{n}\right\}_{1}^{\infty}$ and $\left\{\varphi_{n}\right\}_{-\infty}^{-1}$ orthonormal bases for $\mathfrak{P}$ and for $\mathfrak{Q}$, respectively. As in $\S 4$, we then define

$$
V f=\sum_{-\infty}^{\infty}\left(f, \psi_{n}\right) \varphi_{n}
$$

Here and in what follows a prime on a sum is used to indicate that the term $n=0$ is to be omitted.

Equations (4.1) and (4.4) show that in terms of $V$, we have

$$
\left[T_{P}(A)\right]^{-1} p=A^{-1 / 2} V^{-1} P V A^{-1 / 2} p .
$$

To use this formula, we need an expression for $V^{-1}$, which we shall now derive.

Let $P_{n}$ denote projection on the manifold spanned by the set

$$
\left\{\psi_{k}:-n \leqq k \leqq n, k \neq 0\right\}
$$

For any $f \in \mathfrak{H}, P_{n} f$ of course has the form

$$
P_{n} f=\sum_{k=-n}^{n} \delta_{k, n}(f) \psi_{k} .
$$


As is well known, the coefficients $\delta_{k, n}(f)$ can be computed by means of the formula

$$
\delta_{k, n}(f)=\Delta_{k, n}(f) / \Delta_{n},
$$

where $\Delta_{n}$ is the Gram determinant

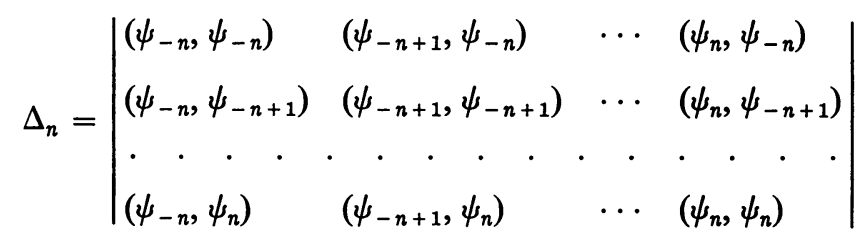

and $\Delta_{k, n}(f)$ is obtained from $\Delta_{n}$ by replacing the $k$ th column of $\Delta_{n}$ by $\left(\left(f, \psi_{-n}\right)\right.$, $\left.\left(f, \psi_{-n+1}\right), \ldots,\left(f, \psi_{n}\right)\right)$. It will be convenient also to define a determinant $D_{k, n}(f)$ obtained from $\Delta_{n}$ by replacing the $k$ th column of $\Delta_{n}$ by $\left(\left(f, \varphi_{-n}\right),\left(f, \varphi_{-n+1}\right), \ldots\right.$, $\left.\left(f, \varphi_{n}\right)\right)$, and to set

$$
d_{k, n}(f)=D_{k, n}(f) / \Delta_{n} .
$$

It should be noted that there are many zeros in the determinants $\Delta_{n}$ and $D_{k, n}(f)$ since $\left(\psi_{j}, \psi_{k}\right)$ is only different from zero when $j=k$ and when $j k<0$. If $p \in \mathfrak{B}$, there are even more zeroes in $D_{k, n}(p)$, since then

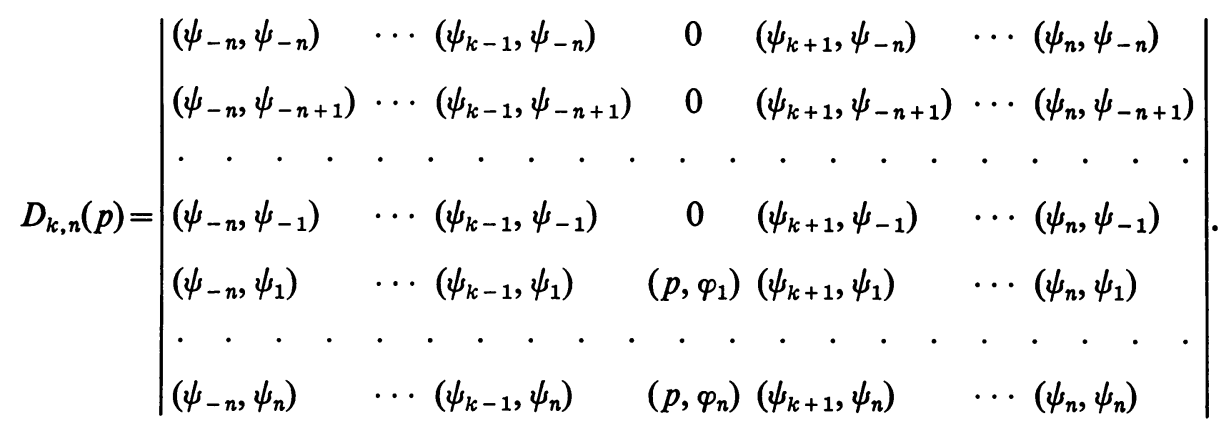

Let $V f=g$. Then, since the set $\left\{\varphi_{n}:-\infty<n<\infty, n \neq 0\right\}$ is orthonormal, we have

Therefore

$$
\left(f, \psi_{n}\right)=\left(g, \varphi_{n}\right), \quad-\infty<n<\infty, n \neq 0 .
$$

$$
\begin{aligned}
P_{n} f & =\sum_{k=-n}^{n} \delta_{k, n}(f) \psi_{k} \\
& =\sum_{k=-n}^{n} d_{k, n}(g) \psi_{k} .
\end{aligned}
$$

If $\left(f, \psi_{n}\right)=0$ for all $n$, then $V f=0$, so that $f=0$. Therefore,

so that

$$
f=\lim _{n \rightarrow \infty} P_{n} f
$$

$$
V^{-1} g=\lim _{n \rightarrow \infty} \sum_{k=-n}^{n} d_{k, n}(g) \psi_{k}
$$

Equations (5.1) and (5.3) can be used in (5.2) to obtain a formula for $\left[T_{P}(A)\right]^{-1}$. 
The result is

$$
\left[T_{P}(A)\right]^{-1} p=\lim _{n \rightarrow \infty} \sum_{k=-n}^{n} d_{k, n}\left(\sum_{j=1}^{\infty}\left(A^{-1 / 2} p, \psi_{j}\right) \varphi_{j}\right) A^{-1 / 2} \psi_{k} .
$$

Obviously,

$$
p^{\prime}=\sum_{j=1}^{\infty}\left(A^{-1 / 2} p, \psi_{j}\right) \varphi_{j}
$$

is an element of $\mathfrak{B}$. Also, when $j \geqq 1,\left(p^{\prime}, \varphi_{j}\right)=\left(A^{-1 / 2} p, \psi_{j}\right)$. Thus, according to the remark made earlier, the coefficients appearing in (5.4) are simply

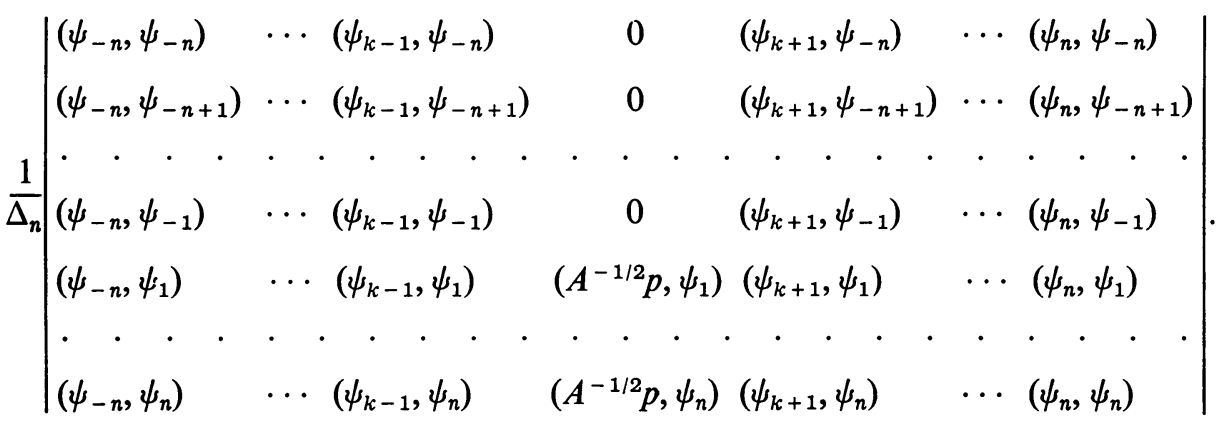

A formula equivalent to (5.4) can also be written in terms of any orthonormal basis for $A^{1 / 2} \mathfrak{B}$. If $\left\{\chi_{k}\right\}_{k=1}^{\infty}$ is any such basis, calculations similar to the ones leading to (5.4) show that an alternative formula is

$$
\left[T_{P}(A)\right]^{-1} p=\lim _{n \rightarrow \infty} \sum_{k=1}^{n} \delta_{k, n}\left(A^{-1 / 2} p\right) A^{-1 / 2} \chi_{k} .
$$

If $A \geqq 0$, the formulas (5.4) and (5.4 bis) become particularly simple. When $A \geqq 0$, we can take $\chi_{k}=\psi_{k}, k=1,2, \ldots$ Also, it is easy to see that the entire sequence $\left\{\psi_{k}:-\infty<k<\infty\right\}$ is orthonormal when $A \geqq 0$. Therefore, an easy calculation shows that

$$
\begin{aligned}
\Delta_{n} & =1, \\
\delta_{k, n}(f) & =\Delta_{k, n}(f) \\
& =\left(f, \psi_{k}\right) .
\end{aligned}
$$

Therefore,

$$
\begin{aligned}
d_{k, n}(f) & =D_{k, n}(f) \\
& =\left(f, \varphi_{k}\right),
\end{aligned}
$$

and

$$
\begin{aligned}
d_{k, n}\left(\sum_{j=1}^{\infty}\left(A^{-1 / 2} p, \psi_{j}\right) \varphi_{j}\right) & =0, & & k<0 \\
& =\left(A^{-1 / 2} p, \psi_{k}\right), & & k>0 .
\end{aligned}
$$


Thus, when $A \geqq 0,(5.4)$ reduces to

$$
\left[T_{P}(A)\right]^{-1} p=\sum_{k=1}^{\infty}\left(A^{-1 / 2} p, \psi_{k}\right) A^{-1 / 2} \psi_{k},
$$

which is equivalent to a formula derived earlier in [12].

(5.4) is a formula for the inverse of $T_{P}(A)$ provided that $A$ has strongly positive real part. It can be considered as supplying a constructive solution of the WienerHopf equation $T_{P}(A) p^{\prime}=p$ provided that one can compute the operators $A^{ \pm 1 / 2}$ and $A_{*}^{ \pm 1 / 2}$. We should like to remark that this is the case whenever $A$ has the form

$$
(A f)(x)=\int_{-\infty}^{\infty} k(x-y) f(y) d y,
$$

whatever the projection $P$ may be. Indeed, if we denote Fourier transforms by addition of the symbol $\uparrow$, we have

$$
(A f)^{\wedge}(\xi)=k^{\wedge}(\xi) f^{\wedge}(\xi)
$$

The condition that $A$ have strongly positive real part is then equivalent to the condition that there exist a $\delta>0$ such that

$$
\operatorname{Re}\left[k^{\wedge}(\xi)\right] \geqq \delta .
$$

In this case, $A^{1 / 2}$ can be computed from the simple formula

$$
\left(A^{1 / 2} f\right)^{\wedge}(\xi)=\left(k^{\wedge}(\xi)\right)^{1 / 2} f^{\wedge}(\xi) .
$$

A similar remark applies to $A^{-1 / 2}$ and to $A_{*}^{ \pm 1 / 2}$.

If $T_{P}(A)$ is invertible, but $A$ does not have strongly positive real part, the proof of Theorem 5 is still constructive and an explicit inversion formula can still in principle be found, but there may be difficulties in the actual computation of some of the terms appearing in it. To understand exactly where the difficulty comes about, we shall derive the inversion formula without the hypothesis that $A$ has strongly positive real part.

The proof of Theorem 5 shows that in this case, the factors $A_{-}$and $A_{+}$are computed by finding an operator $H$ such that $A H$ does have strongly positive real part. This is done by using Theorem 3. Reviewing the proof of Theorem 3, we see that we must begin by factoring $A_{*}$ as in Lemma 1 . This means that we must find an invertible operator $B$ taking $\mathfrak{P}$ onto itself such that $A A_{*}=B_{*} B$. We note that $B$ can be computed explicitly. Indeed, as before, let $\left\{\varphi_{n}\right\}_{1}^{\infty}$ and $\left\{\varphi_{n}\right\}_{-\infty}^{-1}$ be orthonormal bases for $\mathfrak{P}$ and $\mathscr{Q}$ respectively. Let $\left\{\omega_{n}\right\}_{1}^{\infty}$ and $\left\{\omega_{n}\right\}_{-\infty}^{-1}$ be orthonormal bases for $\left(A A_{*}\right)^{1 / 2} \mathfrak{P}$ and $\left(A A_{*}\right)^{-1 / 2} \mathfrak{Q}$, respectively. Then, we have only to define

$$
B f=\left(A A_{*}\right)^{1 / 2} \sum_{-\infty}^{\infty}\left(f, \omega_{n}\right) \varphi_{n}
$$

A simple computation shows that this operator has all the desired properties and, 
of course, it can be computed explicitly if one can compute $\left(A A_{*}\right)^{ \pm 1 / 2}$ explicitly. Of course, we also have

$$
B_{*} f=\sum_{-\infty}^{\infty}\left(\left(A A_{*}\right)^{1 / 2} f, \varphi_{n}\right) \omega_{n}
$$

We now set

$$
U=A^{-1} B_{*}
$$

to obtain the formula $A_{*}=U B$ needed in the proof of Theorem 3. According to the proof of Theorem 3, to find the operator $H$ making $A H$ have strongly positive real part, we must set $H=G B$, where $G$ is the operator associated with $U$ via Theorem 2. According to the proof of Theorem 2, however, $G=P U P+Q U Q$. Since $U$ is computable, $G$ is, and since $G$ is computable, $H$ is. The final result is that the operator

$$
A H=A(P U P+Q U Q) B
$$

has strongly positive real part if $B$ is defined by (5.5) and $U$ by (5.6). Moreover, if we can write $A H=C_{-} C_{+}$, where $C_{-}$and $C_{+}$are invertible, while $C_{-}$takes $\mathfrak{Q}$ onto itself and $C_{+}$takes $\mathfrak{B}$ onto itself, the factors $A_{-}$and $A_{+}$occurring in Theorem 5 can be obtained from the operators $C_{-}$and $C_{+}$by the simple formulas

$$
A_{-}=C_{-}, \quad A_{+}=C_{+} H^{-1} \text {. }
$$

Everything is all right, then, provided only that the operator $A H$ with strongly positive real part can be factored. As we saw in the beginning of this section, this factorization can be performed provided that the operators $(A H)^{ \pm 1 / 2}$ and $(A H)_{*}^{ \pm 1 / 2}$ can be computed explicitly. It is exactly here that a difficulty arises if $A$ does not have strongly positive real part. For, not only does $A$ appear, but also $P$ and $Q$ appear in the definition (5.7) of $A H$. If it occurs that one can find the square roots of $A H$ and $(A H)_{*}$, then indeed an explicit formula for $\left[T_{P}(A)\right]^{-1}$ can be derived. But it is hard to see how these square roots can be computed in general.

6. The finite section theorem. The object of this section is to show that it is possible to obtain an abstract version of the Baxter-Reich "finite section" theorem [1], [11]. We also derive a special version of the theorem that makes clear the role of the various hypotheses used in the general version and which, in addition, is sometimes easier to apply. Finally, we show how the result relates to $T_{\mathrm{P}}(\mathrm{A})$ and to (1.10). The method of proof used in Theorem 7 below is based in part on that used by Devinatz in [4].

As in the rest of the paper, we let $P$ be orthogonal projection onto the subspace $\mathfrak{B}$ of $\mathfrak{H}$. In addition, in this section we suppose that we have a family $\left\{P_{\alpha}\right\}$ of orthogonal projections defined for each $\alpha$ in a directed set $\mathscr{A}$. As usual, we denote the range of $P_{\alpha}$ by $\mathfrak{B}_{\alpha}$ and say that $\left\{P_{\alpha}\right\}$ is a P-family of orthogonal projections if $\mathfrak{P}_{\alpha} \subseteq \mathfrak{P}$ for all $\alpha \in \mathscr{A}$. When $P=\mathrm{P}$, we shall later on take $\mathscr{A}$ to be the positive 
integers and $\mathfrak{P}_{\alpha}$ to be the subspace of $H^{2}$ consisting of those functions whose Fourier coefficients vanish on all integers greater than $\alpha$. In this case, $\left\{P_{\alpha}\right\}$ is clearly a P-family of orthogonal projections.

Let $\left\{P_{\alpha}\right\}$ be any $P$-family of orthogonal projections. We define a set $\mathfrak{B}\left(\left\{P_{\alpha}\right\}\right)$ of operators as follows. A bounded linear operator $B \in \mathfrak{B}\left(\left\{P_{\alpha}\right\}\right)$ if there exists a net $\left\{B_{\alpha}: \alpha \in \mathscr{A}\right\}$ of operators $B_{\alpha}$ with the two properties

$$
\begin{gathered}
B_{\alpha}\left(\mathfrak{P} \ominus \mathfrak{P}_{\alpha}\right) \subseteq \mathfrak{P} \text { for } \alpha \text { large; } \\
B=\lim B_{\alpha} \text { in the operator norm topology. }
\end{gathered}
$$

With this notation, we have

Theorem 7. Let $A$ be a bounded, invertible operator, and let $\left\{P_{\alpha}\right\}$ be a P-family of orthogonal projections. Suppose that $T_{P}(A)$ is invertible and that there exist an $\alpha_{0} \in \mathscr{A}$ and an $m>0$ such that $\alpha \geqq \alpha_{0}$ implies $T_{Q+P_{\alpha}}(A)$ is invertible and

$$
\left\|\left[T_{Q+P_{\alpha}}(A)\right]^{-1}\right\| \leqq m .
$$

Let $\left(^{2}\right) A^{-1}$ and $A_{*}^{-1}$ belong to $\mathfrak{B}\left(\left\{P_{\alpha}\right\}\right)$. Then, there exist an $\alpha_{1} \geqq \alpha_{0}$ and an $M>0$ such that for all $\alpha \geqq \alpha_{1}, T_{P_{\alpha}}(A)$ is invertible and

$$
\left\|\left[T_{P_{\alpha}}(A)\right]^{-1}\right\| \leqq M .
$$

Proof. Let $\alpha \geqq \alpha_{0}$. For $u \in \mathfrak{P}_{\alpha}$, we decompose $A u$ in the form

$$
A u=k+v+h,
$$

where $k \in \mathfrak{Q}, v \in \mathfrak{P}_{\alpha}$, and $h \in \mathfrak{P} \ominus \mathfrak{P}_{\alpha}$. Since $T_{P}(A)$ is invertible, so is $T_{P}\left(A_{*}\right)$; by Corollary 3 , then, $T_{Q}\left(A_{*}^{-1}\right)$ is also invertible. Consequently, by Theorem 3 , there is a bounded, invertible operator $H$ taking $\mathscr{Q}$ onto itself such that

$$
A_{*}^{-1} H+H_{*} A^{-1} \geqq 2 \delta I, \quad \delta>0 .
$$

If we operate on (6.3) by $H_{*} A^{-1}$ and take the inner product with $k$, we find

$$
\left(H_{*} u, k\right)=\left(H_{*} A^{-1} k, k\right)+\left(H_{*} A^{-1} v, k\right)+\left(H_{*} A^{-1} h, k\right) .
$$

Now, $k \in \mathfrak{Q}$, so that $H k \in \mathbb{Q}$ and since $u \in \mathfrak{P}_{\alpha} \subseteq \mathfrak{B}$,

$$
\left(H_{*} u, k\right)=(u, H k)=0 .
$$

Further, if $B_{\alpha} \in \mathfrak{B}\left(\left\{P_{\alpha}\right\}\right)$, we have $\left(B_{\alpha} h, H k\right)=0$. Thus

$$
\left(H_{*} A^{-1} h, k\right)=\left(\left(A^{-1}-B_{\alpha}\right) h, H k\right) .
$$

Consequently, from (6.5) we get

$$
\left|\left(H_{*} A^{-1} k, k\right)\right| \leqq M\left(\|v\|+\left\|A^{-1}-B_{\alpha}\right\| \cdot\|h\|\right)\|k\|,
$$

whenever $M \geqq\left\|H_{*} A^{-1}\right\|+\|H\|$.

${ }^{2}$ ) If the sets $\mathfrak{P}_{\alpha}$ are finite-dimensional, we only need assume $A^{-1} \in \mathfrak{B}\left(\left\{P_{\alpha}\right\}\right)$. 
(6.4) shows that

$$
\left|\left(H_{*} A^{-1} k, k\right)\right| \geqq \operatorname{Re}\left(H_{*} A^{-1} k, k\right) \geqq \delta\|k\|^{2} .
$$

Also, by hypothesis, for every $\varepsilon>0$, there exists an $\alpha_{1} \geqq \alpha_{0}$ such that for every $\alpha \geqq \alpha_{1}$ there is a $B_{\alpha} \in \mathfrak{B}\left(\left\{P_{\alpha}\right\}\right)$ for which $M\left\|A^{-1}-B_{\alpha}\right\| \leqq \varepsilon \delta$. Using these facts in (6.6), we find that there is a constant $M>0$ such that for every $\varepsilon>0$ there exists an $\alpha_{1} \geqq \alpha_{0}$ with the property that whenever $\alpha \geqq \alpha_{1}$,

$$
\|k\| \leqq M\|v\|+\varepsilon\|h\| \text {. }
$$

Next, we make use of the assumption that for $\alpha \geqq \alpha_{0}, T_{Q+P_{\alpha}}(A)$ is uniformly invertible. From Corollary 3 , it follows that $T_{P-P_{\alpha}}\left(A^{-1}\right)$ is invertible, and indeed there is an $m^{\prime}>0$ such that for every $\alpha \geqq \alpha_{0}$,

$$
\left\|T_{P-P_{\alpha}}\left(A^{-1}\right)\right\| \leqq m^{\prime} .
$$

Consequently, Theorem 3 and Corollary 4 show that there exists an $\eta>0$ such that for every $\alpha \geqq \alpha_{0}$ there exists a bounded, invertible operator $H^{\alpha}$ taking $\mathfrak{B} \ominus \mathfrak{P}_{\alpha}$ onto itself and such that

$$
H_{*}^{\alpha} A^{-1}+A_{*}^{-1} H^{\alpha} \geqq 2 \eta I .
$$

We operate on (6.3) by $H_{*}^{\alpha} A^{-1}$ and take the inner product with $h$ to get

$$
\left(H_{*}^{\alpha} u, h\right)=\left(H_{*}^{\alpha} A^{-1} k, h\right)+\left(H_{*}^{\alpha} A^{-1} v, h\right)+\left(H_{*}^{\alpha} A^{-1} h, h\right) .
$$

Since $H^{\alpha} h \in \mathfrak{B} \ominus \mathfrak{P}_{\alpha}$ and $u \in \mathfrak{B}_{\alpha}$, it follows that $\left(H_{*}^{\alpha} u, h\right)=0$. Further, from (6.8), we find that $\left|\left(H_{*}^{\alpha} A^{-1} h, h\right)\right| \geqq \eta\|h\|^{2}$. Using these facts in (6.9), we arrive at the fact that there exists an $M>0$ so large that

$$
\eta\|h\| \leqq M(\|k\|+\|v\|) .
$$

Taking $\varepsilon$ small enough in (6.7) and using (6.7) in (6.10), we find there is an $M>0$ so large that

$$
\|h\| \leqq M\|v\| .
$$

Finally, using (6.7) and (6.11) in (6.3) and noting that $A^{-1}$ is bounded, we see that there exist an $M>0$ and an $\alpha_{1} \in \mathscr{A}$ such that $\alpha \geqq \alpha_{1}$ implies

$$
\|u\| \leqq M\left\|T_{P_{\alpha}}(A) u\right\| \text {. }
$$

This shows that $T_{P_{\alpha}}(A)$ is one-to-one with a closed range.

If the space $\mathfrak{P}_{\alpha}$ is finite-dimensional, (6.12) shows that $T_{P_{\alpha}}(A)$ is invertible. Otherwise, $T_{P}(A)$ and $T_{Q+P_{\alpha}}(A)$ invertible imply that $T_{P}\left(A_{*}\right)$ and $T_{Q+P_{\alpha}}\left(A_{*}\right)$ are invertible. Consequently, we get the inequality (6.12) for $T_{P_{\alpha}}\left(A_{*}\right)=\left[T_{P_{\alpha}}(A)\right]_{*}$. Thus, in any case, $T_{P_{\alpha}}(A)$ is invertible.

We shall now specialize Theorem 7 to obtain a result whose hypotheses are easier to verify and which includes a number of interesting applications. The 
theorem below contains the Baxter-Reich finite section theorem for the circle group as well as a corresponding version for the real line.

As before, we let $\mathscr{A}$ be a directed set, but now we suppose that we have a set $\mathscr{S}=\left\{S^{\alpha}: \alpha \in \mathscr{A}\right\}$ of unitary operators each of which leaves $\mathfrak{P}$ invariant and commutes with $A$. Let us set

$$
\mathfrak{B}_{\alpha}=\mathfrak{B} \ominus\left(S^{\alpha} \mathfrak{P}\right) \text {. }
$$

We suppose that $\mathscr{S}$ has the property $\mathfrak{P}_{\alpha} \subseteq \mathfrak{P}_{\beta}$ if $\alpha \leqq \beta$. Let $\mathfrak{B}_{\alpha}$ be the weak closure of the linear manifold generated by the set $\left\{A_{\beta} S^{\beta}: \beta \in \mathscr{A}\right\} \cup\left\{A_{\beta} S_{*}^{\beta}: \beta \leqq \alpha\right\}$ where $A_{\beta} \mathfrak{P} \subseteq \mathfrak{P}$. We suppose there are nets $\left\{B_{\alpha}: B_{\alpha} \in \mathfrak{B}_{\alpha}, \alpha \in \mathscr{A}\right\}$ and $\left\{C_{\alpha}: C_{\alpha} \in \mathfrak{B}_{\alpha}, \alpha \in \mathscr{A}\right\}$ so that in the operator norm topology,

and $\left({ }^{3}\right)$

$$
A^{-1}=\lim B_{\alpha}
$$

$$
A_{*}^{-1}=\lim C_{\alpha} .
$$

Any triple $(A, \mathscr{S}, \mathfrak{P})$ that satisfies the above conditions will be called a special finite section system.

THEOREM 8. If $(A, \mathscr{S}, \mathfrak{B})$ is a special finite section system, and if $T_{P}(A)$ and $T_{Q}(A)$ are invertible, then there exist an $\alpha_{1} \in \mathscr{A}$ and an $M>0$ such that $\alpha \geqq \alpha_{1}$ implies $T_{P_{\alpha}}(A)$ is invertible and

$$
\left\|\left[T_{P_{\alpha}}(A)\right]^{-1}\right\| \leqq M .
$$

Proof. Since $T_{Q}(A)$ is invertible it follows that $T_{P}\left(A^{-1}\right)$ is invertible. Thus there exists a $\delta>0$ so that

$$
\sup \left\{\left|\left(A^{-1} p, q\right)\right|: p \in \mathfrak{B}, q \in \mathfrak{Q} ;\|p\|=\|q\|=1\right\}=1-\delta .
$$

Since, for every $\alpha \in \mathscr{A}, S^{\alpha}$ is unitary and commutes with $A$, it follows that

$$
\sup \left\{\left|\left(A^{-1} S^{\alpha} p, S^{\alpha} q\right)\right|: p \in \mathfrak{B}, q \in \mathfrak{Q} ;\|p\|=\|q\|=1\right\}=1-\delta .
$$

Since $S^{\alpha}$ commutes with $A$ it also commutes with $A_{*}$ (Fuglede's theorem), and thus we get the same facts for $A_{*}^{-1}$. From Theorem 1 it follows that for every $\alpha \in \mathscr{A}$ the operators $T_{P-P_{\alpha}}\left(A^{-1}\right)$ are invertible, and the inverses have a uniform bound. Consequently for every $\alpha \in \mathscr{A}, T_{Q+P_{\alpha}}(A)$ is invertible and the inverses have a uniform bound. Thus one of the hypotheses of Theorem 7 is satisfied.

Before we proceed to prove the other hypothesis needed in Theorem 7 is valid, we remark that what has just been proved can also be obtained directly from Theorem 3. Indeed, since $T_{P}\left(A^{-1}\right)$ is invertible there is an invertible operator $H$ taking $\mathfrak{B}$ onto itself and a $\delta>0$ so that

$$
A^{-1} H+H_{*} A_{*}^{-1} \geqq \delta I \text {. }
$$

${ }^{3}$ ) If the sets $\mathscr{P}_{\alpha}$ are finite dimensional, this condition on $A_{*}^{-1}$ is not required. 
Now, for every $\alpha \in \mathscr{A}, H^{\alpha}=S^{\alpha} H S_{*}^{\alpha}$ is invertible and takes $\mathfrak{P} \ominus \mathfrak{P}_{\alpha}$ onto itself. Since $S^{\alpha}$ commutes with $A^{-1}$, we get

$$
S^{\alpha}\left(A^{-1} H+H_{*} A_{*}^{-1}\right) S_{*}^{\alpha}=\left(A^{-1} H^{\alpha}+H_{*}^{\alpha} A_{*}^{-1}\right) \geqq \delta I .
$$

Thus, Theorem 3 and Corollary 4 tell us that $T_{P-P_{\alpha}}\left(A^{-1}\right)$ is invertible for every $\alpha$ and that its inverse has a bound independent of $\alpha$. Consequently, the same is true of the operator $T_{Q+P_{\alpha}}(A)$.

To show that the other hypothesis of Theorem 7 is satisfied we first remark that if $\beta \leqq \alpha$, then

$$
S_{*}^{\beta}\left(\mathfrak{P} \ominus \mathfrak{P}_{\alpha}\right) \subseteq S_{*}^{\beta}\left(\mathfrak{P} \ominus \mathfrak{P}_{\beta}\right)=\mathfrak{P},
$$

since $S^{\beta}$ is unitary. Also,

$$
S^{\beta}\left(\mathfrak{P} \ominus \mathfrak{P}_{\alpha}\right) \subseteq \mathfrak{P}
$$

for every $\beta \in \mathscr{A}$, and it follows that each operator in $\mathfrak{B}_{\alpha}$ takes $\mathfrak{B} \ominus \mathfrak{P}_{\alpha}$ into $\mathfrak{B}$. Since $A^{-1}$ and $A_{*}^{-1}$ are limits of nets in $\mathfrak{B}_{\alpha}$ it follows that all of the hypotheses of Theorem 7 are satisfied. Thus, Theorem 8 is established.

We wish to apply Theorem 8 to certain Toeplitz operators on $H^{2}$ spaces. To do so, we introduce some terminology. We let $\mathfrak{S}$ be a separable Hilbert space, $L^{2}(\mathfrak{E})$ the space of (equivalence classes of) $\mathfrak{S}$-valued, weakly measurable functions on the circle group which are square summable, $H^{2}(\mathfrak{S})$ the subspace of $L^{2}(\mathfrak{S})$ consisting of those elements whose Fourier coefficients vanish on the negative integers, and $K^{2}(\mathfrak{E})$ the subspace of $L^{2}(\mathfrak{S})$ consisting of the elements whose Fourier coefficients vanish on the positive integers. If $\mathfrak{B}$ is the set of bounded operators on $\mathfrak{F}$, then we can form the spaces $L^{2}(\mathfrak{B})$ and $H^{2}(\mathfrak{B})$ in a manner similar to the formation of the spaces $L^{2}(\mathfrak{S})$ and $H^{2}(\mathfrak{S})$. Also, we define $H^{\infty}(\mathfrak{B})$ as the subspace of $H^{2}(\mathfrak{B})$ consisting of essentially bounded elements.

If $A(\theta)$ is a weakly measurable, essentially bounded, $\mathfrak{B}$-valued function on the circle group, we define an operator $A$ on $L^{2}(\mathfrak{S})$ by the formula

$$
(A f)(\theta)=A(\theta) f(\theta),
$$

and two Toeplitz operators by

$$
\begin{aligned}
& T^{+}(A)=\mathrm{P}^{+} A \mid H^{2}(\mathfrak{S}), \\
& T^{-}(A)=\mathrm{P}^{-} A \mid K^{2}(\mathfrak{S}),
\end{aligned}
$$

where $\mathrm{P}^{+}$is the projection of $L^{2}(\mathfrak{S})$ onto $H^{2}(\mathfrak{S})$, and $\mathrm{P}^{-}$is the projection of $L^{2}(\mathfrak{S})$ onto $K^{2}(\mathfrak{S})$. We have changed the notation from $\mathrm{P}$ to $\mathrm{P}^{+}$for the sake of symmetry.

For every nonnegative integer $n$, let $\mathfrak{P}_{n}$ be the set of all $\mathfrak{S}$-valued trigonometric polynomials of the form

$$
\sum_{k=0}^{n} e^{i k \theta} x_{k}, \quad x_{k} \in \mathfrak{S}
$$


If $P_{n}$ is the projection onto $\mathfrak{B}_{n}$, then we define

$$
T_{n}^{+}(A)=P_{n} T^{+}(A) \mid \mathfrak{B}_{n} .
$$

Denote by $C+H^{\infty}$ the closure in $L^{\infty}(\mathfrak{B})$ of those elements whose Fourier coefficients vanish on all but a finite number of negative integers. (The reason for the notation is that if $\mathfrak{F}$ is one-dimensional, it was shown in [8] that $C+H^{\infty}$ is the vector sum of $H^{\infty}(\mathfrak{S})$ and the continuous functions $C$.)

COROLlaRy 8. Suppose $A(\theta)$ is a weakly measurable, essentially bounded, $\mathfrak{B}$-valued function on the circle group, invertible a.e., and whose inverse, $A^{-1}(\theta)$, is an element of $C+H^{\infty}$. A necessary and sufficient condition that $T^{+}(A)$ and $T^{-}(A)$ be invertible is that there exist an $n_{0}$ and an $M>0$ so that for all $n \geqq n_{0}, T_{n}^{+}(A)$ is invertible and $\left\|\left[T_{n}^{+}(A)\right]^{-1}\right\| \leqq M$.

Proof. Define a unitary operator $S$ on $L^{2}(H)$ by

$$
S g(\theta)=e^{i \theta} g(\theta),
$$

and take $\mathscr{S}=\left\{S^{n}: n \geqq 0\right\}$. From the hypotheses it follows that $\left(A, \mathscr{S}, H^{2}(\mathfrak{S})\right)$ is a special finite section system except that we have made no assumption about $A_{*}^{-1}(\theta)$. However, the proofs of Theorems 7 and 8 show that there exists an $M>0$ so that for all sufficiently large $n$, and for every $f \in \mathfrak{B}_{n}$

$$
\|f\| \leqq M\left\|T_{n}^{+}(A) f\right\| .
$$

If we work with the space $K^{2}(\mathfrak{S})$ and $A_{*}(\theta)$ it follows by the same proofs as used in Theorems 7 and 8 and the hypothesis on $A^{-1}(\theta)$ that there exists an $M>0$ so that for all sufficiently large $n$ and for every $f \in K_{n}^{2}(\mathfrak{S})$ we have

$$
\|f\| \leqq M\left\|T_{n}^{-}\left(A_{*}\right) f\right\| .
$$

Now for every nonnegative integer $n$ and every $B \in \mathfrak{B}$, a straightforward calculation shows that $S_{*}^{n}$ takes $H_{n}^{2}(\mathfrak{S})$ onto $K_{n}^{2}(\mathfrak{S})$ and for every $g \in H_{n}^{2}(\mathfrak{S})$ we have

$$
S^{n} T_{n}^{-}(B) S_{* g}^{n} g=T_{n}^{+}(B) g .
$$

In particular if we use $A_{*}$ for $B$ in (6.15), then from (6.14) it follows that an inequality such as (6.13) is true when $A$ is replaced by $A_{*}$. This proves the necessity.

To prove the sufficiency, we suppose that there exists an $M>0$ so that for all sufficiently large $n, T_{n}^{+}(A)$ is invertible and $\left\|\left[T_{n}^{+}(A)\right]^{-1}\right\| \leqq M$. This means that for $f \in \mathfrak{P}_{n}$ and for every $m \geqq n$,

$$
\|f\| \leqq M\left\|T_{m}^{+}(A) f\right\| .
$$

By letting $m \rightarrow \infty$ we see this inequality persists for $T^{+}(A)$. Since $P_{n} \rightarrow I$ as $n \rightarrow \infty$, it follows that $T^{+}(A)$ is one-to-one with a closed range. Now, $\left[T_{n}^{+}(A)\right]_{*}=T_{n}^{+}\left(A_{*}\right)$ and thus for all sufficiently large $n, T_{n}^{+}\left(A_{*}\right)$ is invertible and $\left\|\left[T_{n}^{+}\left(A_{*}\right)\right]^{-1}\right\|$ $=\left\|\left[T_{n}^{+}(A)\right]^{-1}\right\| \leqq M$. Using the same argument as above it follows that $T^{+}\left(A_{*}\right)$ is 
one-to-one with a closed range. Since the range of $T^{+}(A)$ is the orthogonal complement of the nullspace of $T^{+}\left(A_{*}\right)$, we see that the range of $T^{+}(A)$ is all of $H^{2}(\mathfrak{S})$. Thus $T^{+}(A)$ is invertible.

To complete the proof of sufficiency it remains to establish that $T^{-}(A)$ is invertible. However, by using (6.3) with $B$ replaced by $A$ and then $A_{*}$, it follows by the same argument as above that $T^{-}(A)$ is invertible. The proof is complete.

If $\mathfrak{S}$ is one-dimensional, then $T^{+}(A)$ being invertible implies $T^{-}(A)$ is invertible. Indeed, there exists a bounded function $H$ in $H^{\infty}$ so that $1 / H$ is in $H^{\infty}$ and $A H+H_{*} A_{*} \geqq \delta$. But $A$ and $H$ being complex valued means they are permutable so that $H A+A_{*} H_{*} \geqq \delta$. Also, $H_{*}$ is in $K^{\infty}$, so that $T^{-}\left(A_{*}\right)$ is invertible. Thus, $T^{-}(A)$ is invertible. Whether or not this result persists when the dimension of $\mathfrak{S E}$ is greater than 1 is not known.

As another special case we consider the question of a finite section theorem when the spaces are defined on the real line instead of the circle group. In this case, $L^{2}(\mathfrak{S})$ is the Lebesgue space of (equivalence classes of ) square integrable $\mathfrak{S g}$-valued functions defined on the real line, $H^{2}(\mathfrak{S})$ is the subspace consisting of those elements whose Fourier transforms vanish on the negative real numbers, and $K^{2}(\mathfrak{E})$ is the subspace consisting of those functions whose Fourier transforms vanish on the positive real numbers. In a similar manner as for the circle group we may define the spaces $L^{2}(\mathfrak{B}), H^{2}(\mathfrak{B})$ and $H^{\infty}(\mathfrak{B})$. Also, if $A(x)$ is an essentially bounded, weakly measurable, $\mathfrak{B}$-valued function defined on the real line, it is clear how to define the operators $T^{+}(A)$ and $T^{-}(A)$.

Let $r$ be a positive real number, $\mathfrak{P}_{r}$ the subspace of $H^{2}(\mathfrak{S})$ consisting of those elements whose Fourier transforms vanish outside of the interval $[0, r]$, and $P_{r}$ the projection onto $\mathfrak{P}_{r}$. We define

$$
T_{r}^{+}(A)=P_{r} T^{+}(A) \mid \mathfrak{P}_{r} .
$$

The next result can be proved by using the Cayley transform as indicated in $\S 1$. However, we give a proof based directly on Theorem 8 .

COROLlary 9. Suppose $A(x)$ is a bounded $\mathfrak{B}$-valued function defined on the real line whose inverse, $A^{-1}(x)$, is a bounded uniformly continuous (in the operator norm of $\mathfrak{B})$ function. A necessary and sufficient condition that $T^{+}(A)$ and $T^{-}(A)$ be invertible is that there exist an $r_{0} \geqq 0$ and an $M>0$ so that for all $r \geqq r_{0}, T_{r}^{+}(A)$ is invertible and $\left\|\left[T_{r}^{+}(A)\right]^{-1}\right\| \leqq M$.

Proof. For every $f \in L^{2}(\mathfrak{S})$ and for every real $t$, we define

$$
S^{t} f(x)=e^{i t x} f(x)
$$

and $\mathscr{S}=\left\{S^{t}: t \geqq 0\right\}$. Clearly, $S_{*}^{t}=S^{-t}$, and $\mathfrak{P}_{r}=H^{2}(\mathfrak{E}) \ominus S^{r} H^{2}(\mathfrak{E})$ for $r>0$.

Now, since $A^{-1}$ is bounded and uniformly continuous the same is true for $A_{*}^{-1}$. Consequently, using the $\mathfrak{B}$-valued version of the Fejer approximation theorem for the real line, we find that there are nets of operators $\left\{B_{r}: r \geqq 0\right\}$ and $\left\{C_{r}: r \geqq 0\right\}$, 
each operator $B_{r}$ and $C_{r}$ being in the weak closure of the linear manifold generated by the set $\left\{B_{t} S^{t}:-r \leqq t \leqq r\right\}$, where $B_{t}$ is to be identified with an element of $\mathfrak{B}$, and so that

$$
\begin{aligned}
& A^{-1}=\lim _{r \rightarrow \infty} B_{r}, \\
& A_{*}^{-1}=\lim _{r \rightarrow \infty} C_{r},
\end{aligned}
$$

where the convergence is in the operator norm. Thus we see that $\left(A, \mathscr{S}, H^{2}(\mathfrak{Z})\right)$ is a special finite section system. The necessity now follows from Theorem 8 .

The sufficiency is proved in exactly the same manner as the sufficiency of Corollary 8.

Finally, we should like to remark that the proof of Corollary 8 can also be obtained using the Pousson-Rabindranathan results mentioned in $\$ 1$. Using the notation introduced just before Corollary 7 , these authors prove first of all that $T^{+}(A)$ is invertible if and only if a.e. $A(\theta)=U(\theta) B(\theta)$, where $B(\theta)$ and $B(\theta)^{-1}$ are in $H^{\infty}(\mathfrak{B})$ and $U(\theta)$ is a unitary valued operator function which satisfies $\|U-G\|_{\infty}$ $<1$, where $G(\theta)$ and $G(\theta)^{-1}$ are operator-valued functions in $H^{\infty}(\mathfrak{B}),\|U-G\|_{\infty}$ $=$ ess sup $\|U(\theta)-G(\theta)\|$, and $\|\cdot\|$ is the operator norm in $\mathfrak{B}$.

Except for the considerable complications caused by working on an infinitedimensional space, the method used by Rabindranathan is the same as that of Pousson. Both authors first dualize an extremum problem as in the paper by Helson and Szegö [7] and then get the result mentioned from this. This is essentially the same program as carried out in [3] for numerical valued functions.

Using the same technique which we used in the proof of Theorem 3 we can push their result a little further to obtain the following.

If $A(\theta)$ is a weakly measurable, essentially bounded $\mathfrak{B}$-valued function, then $T^{+}(A)$ is invertible if and only if there exists an invertible element $H \in H^{\infty}(\mathfrak{B})$ and $a \delta>0$ so that a.e.

$$
A(\theta) H(\theta)+H(\theta)_{*} A(\theta)_{*} \geqq \delta I,
$$

where I is the identity on $\mathfrak{H}$.

Of course, we can get a similar result for $T^{-}(A)$.

To see how this can be used to get the proof of Corollary 8 , we simply notice that $H$ acting as an operator on $H^{2}(\mathfrak{Y})$ takes $H^{2}(\mathfrak{S}) \ominus \mathfrak{B}_{n}$ onto itself, so that by Theorem 3 it follows that $T_{\mathrm{P}^{+}{ }_{-} P_{n}}\left(A^{-1}\right)$ is invertible and the inverses have a uniform bound. The proof then proceeds as before. The reason that a set of unitary operators does not seem to enter into this proof is that the relevant set of unitary operators for Theorem 7 is the set of operators obtained by multiplication by characters of the circle group, which permute with $H$. Thus if $S$ is the operator obtained by multiplication by $\exp (i \theta)$, we have $S^{n} H S_{*}^{n}=H$.

\section{REFERENCES}

1. G. Baxter, A norm inequality for a "finite section" Wiener-Hopf equation, Illinois J. Math. 7 (1963), 97-103. 
2. A. Beurling, On two problems concerning linear transformations in Hilbert space, Acta Math. 81 (1949), 239-255.

3. A. Devinatz, Toeplitz operators on $H^{2}$ spaces, Trans. Amer. Math. Soc. 112 (1964), 304-317.

4. - An extension of a limit theorem of G. Szegö, J. Math. Anal. Appl. 14 (1966), 499-510.

5. - On Wiener-Hopf operators, Proceedings Irvine Conference on Functional Analysis, Thompson, Washington, D. C., 1967, pp. 81-118.

6. P. Hartman and A. Wintner, On the spectra of Toeplitz's matrices, Amer. J. Math. 72 (1950), 359-366.

7. H. Helson and G. Szegö, A problem in prediction theory, Ann. Mat. Pura Appl. 51 (1960), 107-138.

8. H. Helson and D. Sarason, Past and future, Math. Scand. 21 (1967), 5-16.

9. H. R. Pousson, Systems of Toeplitz operators on $H^{2}$. I, II, Proc. Amer. Math. Soc. 19 (1968), 603-608.

10. M. Rabindranathan, Generalized Toeplitz operators, Thesis, University of California, Berkeley, 1968.

11. E. Reich, On non-Hermitian Toeplitz matrices, Math. Scand. 10 (1962), 145-152.

12. M. Shinbrot, On singular integral operators, J. Math. Mech. 13 (1964), 395-406.

13. - On the range of general Wiener-Hopf operators, J. Math. Mech. 18 (1969), 587-602.

14. - The solution of some integral equations of Wiener-Hopf type, Quart. Appl. Math. (to appear).

15. N. Wiener and E. Hopf, Über eine Klasse singulärer Integralgleichungen. S.-B. Preuss. Akad. Wiss. (1931), 696-706.

16. H. Widom, Inversion of Toeplitz matrices. II, Illinois J. Math. 4 (1960), 88-99.

17. - Inversion of Toeplitz matrices. III, Abstract 564-246, Notices Amer. Math. Soc. 7 (1960), 63.

18. A. Brown and P. Halmos, Algebraic properties of Toeplitz operators, J. Reine Angew. Math. 213 (1963), 89-102.

\section{NORTHWESTERN UNIVERSITY, EVANSTON, ILLINOIS}

